\title{
Design of Temperature and Humidity Data Acquisition System
}

\section{Based on ARM}

\author{
Pei-xue Liu ${ }^{1, a}$, Yu-jie Chen ${ }^{1, b}$, Bao-hua Jiang ${ }^{1, c}$, Shu-mei Liu ${ }^{1, d}$ \\ Qingdao Huanghai College, Qingdao 266427, China \\ a93987898@qq.com, c $1073578901 @ q q . c o m$ \\ b chenyujie_cc@163.com, ${ }^{\text {d }} 1309889426 @ q q . c o m$
}

Keywords: STM32, ZigBee, Temperature and humidity, Delphi7

\begin{abstract}
With the development of science and technology, the industry demand for temperature and humidity is increasing day by day. In this paper, a design of temperature and humidity collection system based on ARM is put forward. The system is designed by combining a ZigBee technology and GSM technology with STM32 as a processor to receive data on the temperature and humidity collection system, real-time through the host computer and mobile terminal to view the data. The hardware and software design of the system is introduced in detail, and the system is tested. The test results show that the system works well and can meet the needs of GSP certification and other temperature and humidity.
\end{abstract}

\section{Introduction}

The temperature and humidity acquisition system collects system via SMS way will monitor the data sent to the mobile monitoring terminal, control center through GSM module in TCP/IP way communicate with PC, control center can be carried out on the data analysis and processing and remote control. The system can distribute multi-point data of temperature and humidity, and can realize the temperature and humidity data at any time, the collection and control of the equipment. Compared with the traditional model, the system not only has high efficiency and adequate resource utilization, but also has short period of temperature and humidity measurement and low cost. The system adopts emerging technologies, which can avoid accidents, and the system is simple, reliable and low cost. Therefore, the system has very important market application value.

\section{Overall System Design}

The system is divided into five parts: data acquisition terminal, control center, mobile phone terminal, background server software, user client software, etc., and system work block diagram.

\section{System hardware design and ZigBee technology}

\section{ZigBee technology}

Wireless technology rapid development, network requirement enhances unceasingly, adapted to the demand of standardized people also gradually appear in front of the wireless 
technology, all kinds of standard has been created. Currently, wi-fi technology, bluetooth, ZigBee technology and other short-range wireless communication technologies. Comparison table (see table 1 and Fig.1).

Table 1 ZigBee and other standard comparison tables

\begin{tabular}{|c|c|c|c|}
\hline specification & ZigBee & bluetooth & Wi-Fi \\
\hline & $\begin{array}{c}868 / 15 \mathrm{MHz} / 2.4 \mathrm{G} \\
\mathrm{Hz}\end{array}$ & $2.4 \mathrm{GHz}$ & $2.4 \mathrm{GHz}$ \\
\hline Connection device & $2^{16} \sim 2^{64}$ & 7 & 255 \\
\hline Maximum consumption & $1 \sim 3 \mathrm{~mW}$ & $1-100 \mathrm{~mW}$ & $100 \mathrm{~mW}$ \\
\hline Transmission rate & $20 \sim 250 \mathrm{Kbps}$ & $1 \mathrm{Mbps}$ & $11 \mathrm{Mbps}$ \\
\hline Main application & $\begin{array}{l}\text { control network } \\
\text { sensor network }\end{array}$ & personal network & $\begin{array}{l}\text { Wireless Local } \\
\text { Area Network }\end{array}$ \\
\hline
\end{tabular}

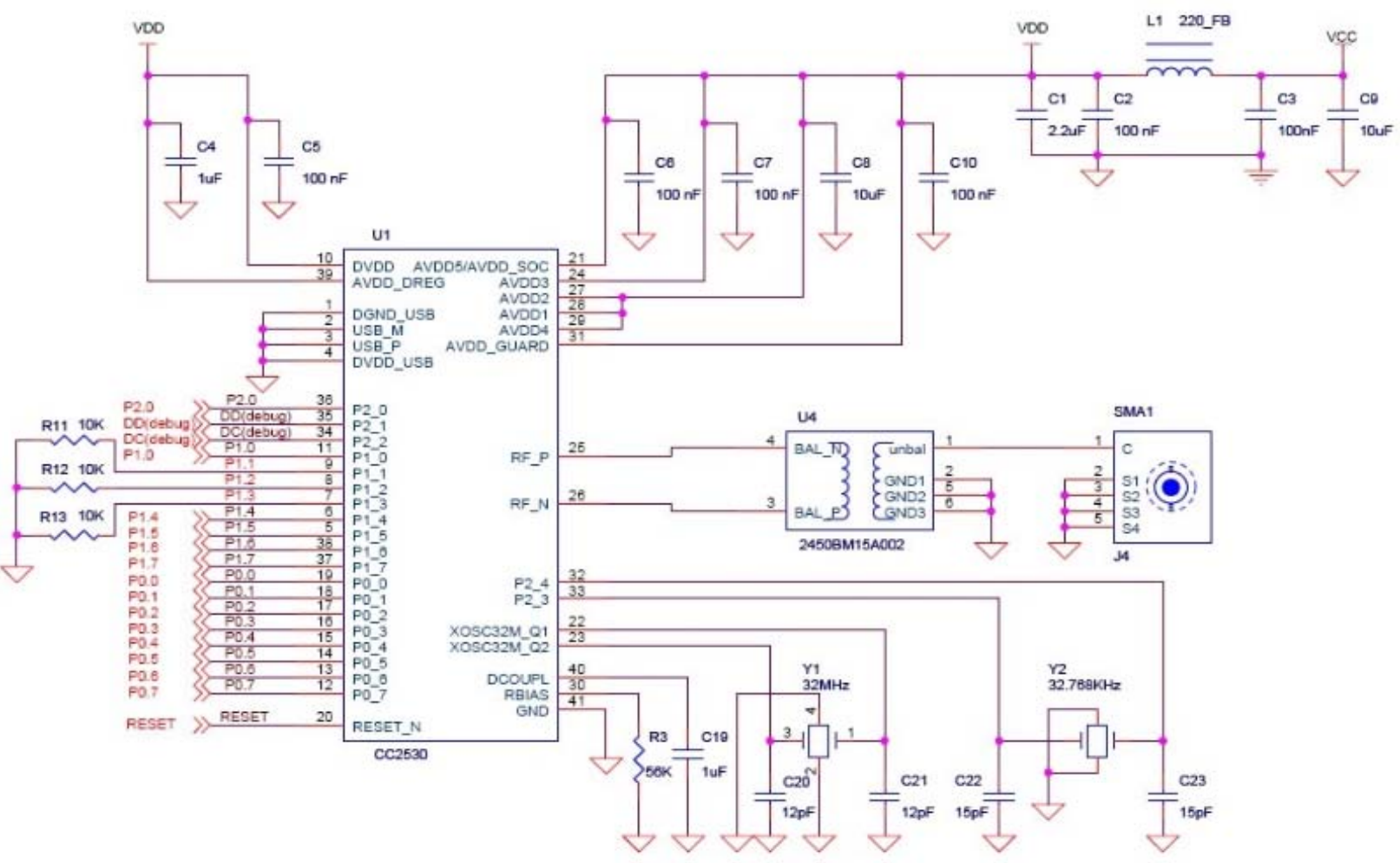

Figure 1 Circuit design of control center

The control center is designed to use the STM32 series of kernel processors. The kernel architecture based on advanced M3, STM32 microcontroller series on the flash memory size up to $512 \mathrm{~KB}$, more equipped with large capacity SRAM, USES has the higher efficiency of instruction and stronger Tumb - 2 instruction set, the performance of single chip microcomputer is also provides a display, storage, voice, and advanced control and low power 
consumption superior performance with a variety of power saving mode, low power consumption is particularly suitable for industrial control products and all kinds of consumer electronics. Compared with traditional single chip microprocessors, the STM32 series has the following advantages:

(1) framework of cortex-m3 kernel.The harvard processor structure, which has both the command and the data to perform at the same time;The built-in interrupt controller responds quickly, and the 32-bit kernel ensures the efficient operation of its speed.Its performance has been greatly improved by its characteristics such as high real time, short low power consumption and time-delay time.

(2) excellent efficiency of power consumption.Microprocessor STM32 series has the function of dynamic power consumption in run mode, its low standby power consumption and low voltage (battery) ability to work, make the user to enjoy high performance at the same time, also can enjoy the low power consumption.

(3) high internal integration.The complexity of the development program is greatly reduced by the rich resources such as door dog timer, RC oscillator circuit, 16 PWM synchronization, ADC sampling channel, AC timer, power controller and so on.

\section{Software design}

Control center equipment open after the first hardware initialization, then UCOSII system initialization, and then establish a UCOSII system startup tasks, and in the startup tasks to registration of work tasks, each task is essentially a cycle, delay this task in the design are all over itself to yield the CPU control, so that to reach the effect of task switching between operation.

Five mission control center is a major operation, the main task is responsible for the page display and set the parameters of a task is responsible for the power state of query, SIM card and the way of inquiry, power supply testing signal, task 2 is responsible for the relay loop testing, alarm, send SMS, task 3 is responsible for the communication between control center and the client upload data and parameters change, task 4 is responsible for the SMS command receiving and the data regularly send text messages. Control center program block diagram (see figure 2) . 


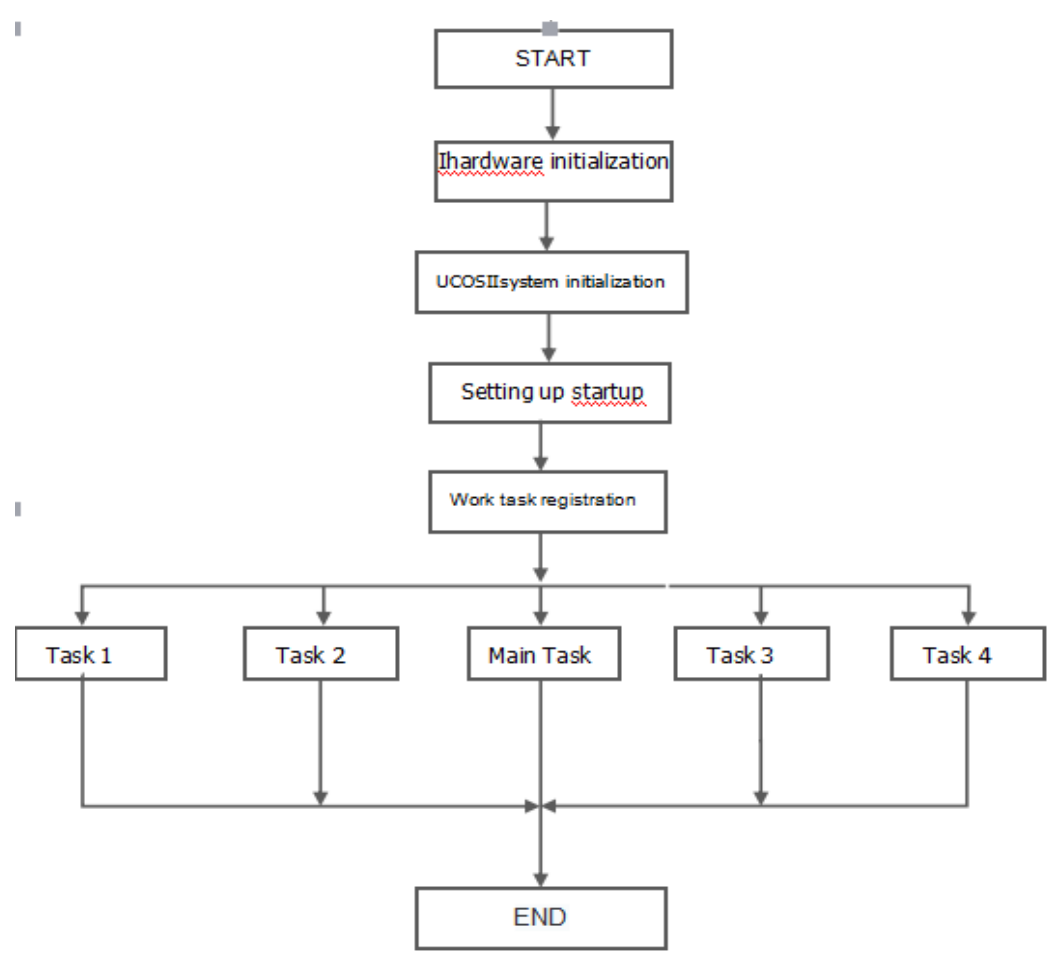

Figure 2 Program block diagram

\section{System Test}

The system normally runs through ZigBee data acquisition terminal node and control center ZigBee coordinator group network and the control center GSM module establishes the TCP connection with the upper computer. The terminal node is responsible for the normal operation, the temperature and humidity data acquisition, will be sent to the data collected by ZigBee network control center on the ZigBee coordinator, the coordinator is responsible for the data received via a serial port is forwarded to the control center of the processor, the processor through GSM module to establish TCP link to send data to a backend server, a backend server to forward the data to corresponding user client, user client upload real-time display of temperature and humidity data. Normal operation diagram of the system.

\section{Conclusion}

The purpose of this system design a GSP authentication of the temperature and humidity data acquisition system, the system hardware to complete function: mobile phone to send text messages to users, +0.5 , the temperature and humidity error alarm, the alarm limit can be modified, with four-way control relay, can be mass record data and interval can be modified, but through the SD card system upgrade, touch screen operation, password protection function.

In the process of completing graduation design, I have not only consolidated what I learned before, but also studied the knowledge that I had not previously been exposed to. I learned the ZigBee network structures, and the data transceiver, the GSM communication, and deepened the understanding of UCOSII system, and for the development of the page can be more skilled use UCGUI, developed the page more beautiful and practical, also learned to develop the upper machine PC development language Delphi, preliminary understanding the Delphi 
powerful database development capabilities, have a further understanding on the Delphi language development.

\section{Acknowledgement}

This research was financially supported by the College Science and technology project of Shandong Province (J15LN59, J16LN75 ,J16LN78)and QingDao huanghai unversity project(2016kj04).

\section{References}

[1] wu yizhen. Selection of cold and heat source solutions for building energy efficient air-conditioning [J]. Energy saving, 2007, 6 (2) : 51-53.

[2] bending, Chen liping, wang. Research on the communication of 0C/os-iii mission [J]. Computer knowledge and technology, 2012, (2) : 306-308.

[3] wang bin, cheng xue. Design of an ARM based greenhouse data acquisition system [J]. Anhui agricultural science. 2011 (12).

[4] wang wuli, Yang hua. Design of grain warehouse temperature and humidity measurement and control system based on SHT11. [J]. Instrumentation technology and sensors, 2010 (9).

[5] feisi research product research center. Delphi database application development [M]. Beijing: electronics industry press, 2003. 